S.D. Ryder, D.J. Pisano, M.A. Walker, and K.C. Freeman, eds.

\title{
Molecular hydrogen as dark mass in dwarf galaxies
}

\author{
P. R. Williams \\ Shanghai Astronomical Observatory, Chinese Academy of Sciences, 80 \\ Nandan Road, Shanghai 200030, PR China \\ C. Marzok, S. Myers, A. H. Nelson \\ Department of Physics and Astronomy, Cardiff University, Cardiff CF2 \\ $3 Y B$, Wales, UK
}

\begin{abstract}
We report here an example of a series of computer simulations of the formation of disk galaxies, including a standard cold dark matter component, gas, and star formation, which result in objects which closely resemble observed giant galaxies, with a population of long-lived dwarf satellites which contain little or no non-baryonic dark matter.
\end{abstract}

There are two generations of dwarfs distinguishable during the formation of the example galaxy: 1) A primordial generation of dwarfs, each with a dark matter halo, which form in the fragmentation of the collapsing protogalaxy by the Jean's instability. These galaxies merge, forming a substantial part of the main galaxy's disk, and do not survive to $z=0 ; 2$ ) A second generation of dwarfs which at a later stage condense out of the unprocessed primordial material with enhanced gas density surrounding the giant, and have sufficiently high angular momentum orbits to avoid merging up to $z=0$ (see Figure 1). These second generation dwarfs reproduce the properties of observed Local Group dwarfs with respect to mass, star formation history, morphology, and kinematics, except for their gas mass. They do not have individual dark matter halos, but orbit in the more spherical dark matter halo of the giant galaxy. If the cold dark matter were to be made sufficiently dissipative for it to settle in the gravitational wells of the dwarfs, it would also form a significant component of the giant galaxy's disk, contrary to recent observational estimates that the disk of the Milky Way has no significant dark matter component.

However the dwarfs do have an excess of gas in comparison to the amounts so far observed in dwarf galaxies, resulting in both their kinematics and total masses being consistent with observations. This suggests that the dark matter component of at least some dwarfs is simply, difficult to detect, molecular hydrogen. Some recent observational evidence supports this suggestion: in lowmetallicity dwarfs the $\mathrm{CO}$ to $\mathrm{H}_{2}$ conversion ratio could be up to 100 times higher than in the Milky Way (Lisenfeld et al. 2002; Papadopoulos et al. 2002); in tidal dwarf galaxies formed from evolved material the detection of $\mathrm{H}_{2}$ by $\mathrm{CO}$ indicates that there is sufficient $\mathrm{H}_{2}$ to bind these galaxies without non-baryonic dark matter (Braine et al. 2001); analysis of the pressure confinement of dwarf galaxy HII regions suggests the $\mathrm{H}_{2}$ may be more abundant than previously thought 

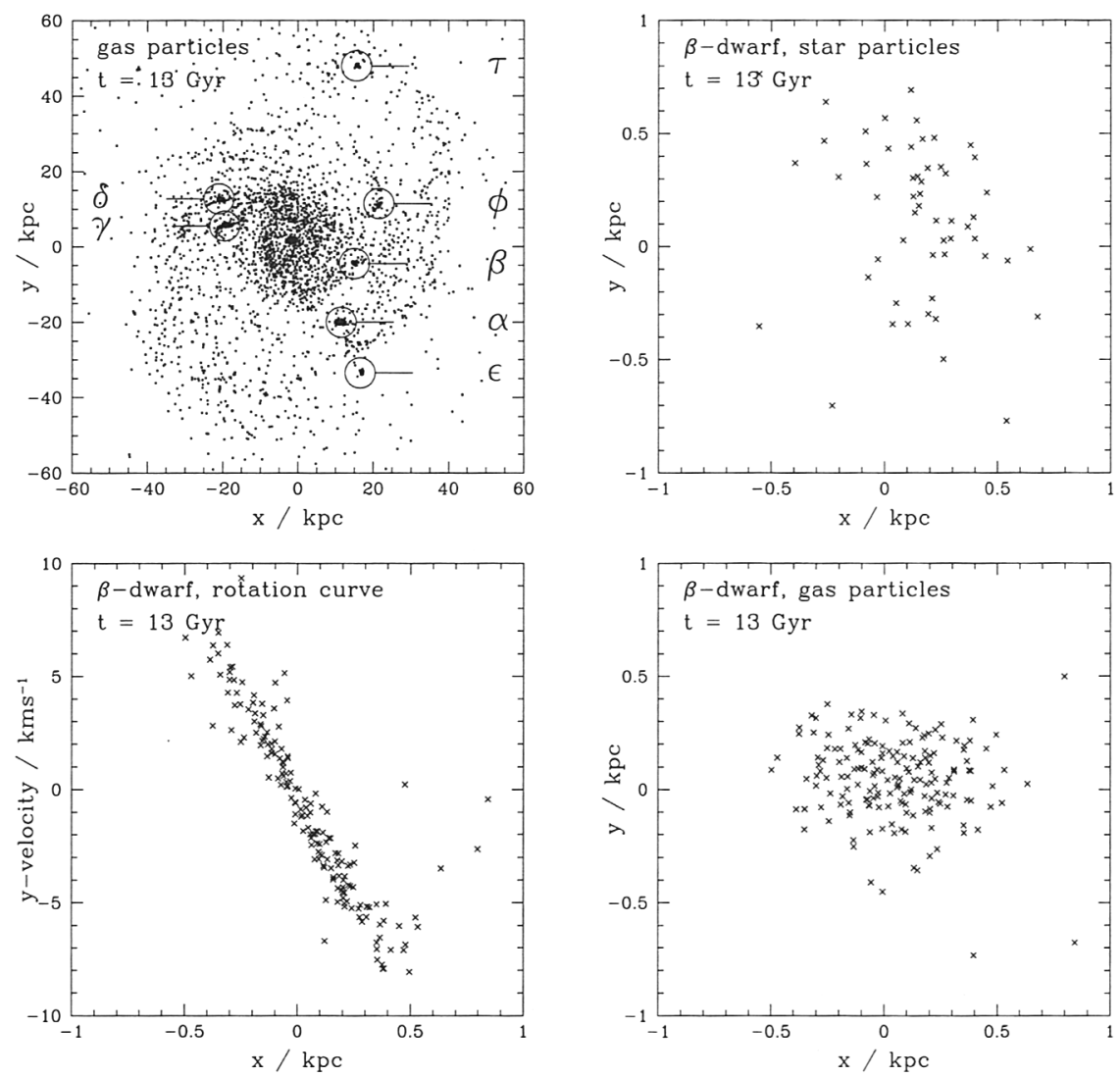

Figure 1. The $\beta$-dwarf galaxy formed at $t \simeq 7 \mathrm{Gyr}$, and subsequently orbited the main galaxy 7 times. Its most recent burst of star formation was at $t \simeq 11 \mathrm{Gyr}$, when it interacted strongly with two of the other dwarfs (peak SFR 0.2 $\mathrm{M}_{\odot} \mathrm{yr}^{-1}$ ). For full simulation details see Williams \& Nelson (2001) and www.astro.cf.ac.uk/pub/Alistair.Nelson/sim8.

(Elmegreen \& Hunter 2000); and there has been direct detection of massive amounts of $\mathrm{H}_{2}$ in NGC891 (Valentijn \& van der Werf 1999).

\section{References}

Braine, J., et al., A\&A, 2001, 378, 51

Elmegreen, B. G., Hunter, D. A., 2000, ApJ, 540, 814

Lisenfeld, U., et al., 2002, A\&A, 382, 860

Papadopoulos, P. P., et al., 2002, ApJ, 579, 270

Valentijn, E. A., van der Werf, P. P., 1999, ApJ, 522, L29

Williams, P. R., Nelson, A. H., 2001, A\&A, 374, 839 